STOMACH

\title{
Helicobacter pylori infection modifies gastric and plasma ghrelin dynamics in Mongolian gerbils
}

\author{
H Suzuki, T Masaoka, H Hosoda, T Ota, Y Minegishi, S Nomura, K Kangawa, H Ishii
}

Gut 2004;53:187-194

See end of article for authors' affiliations

.....................

Correspondence to:

Dr H Suzuki, Department

of Internal Medicine,

School of Medicine, Keio

University, 35

Shinanomachi, Shinjuku-

ku, Tokyo 160-8582,

Japan;

hsuzuki@sc.itc.keio.ac.jp

Accepted for publication

2 September 2003

\begin{abstract}
Background and aim: Although ghrelin, a novel growth hormone releasing peptide localised mainly in the gastric fundus, is reported not only to accelerate food passage and gastrointestinal motility but also to affect appetite and weight control, regulation of gastric ghrelin secretion under the conditions of gastric Helicobacter pylori infection is unknown. The present study was designed to investigate plasma and gastric ghrelin levels in Mongolian gerbils with $\mathrm{H}$ pylori colonisation of the gastric mucosa.

Methods: Gerbils orally inoculated with $H$ pylori were examined after inoculation. To examine preproghrelin mRNA expression in the gastric mucosa, cDNA encoding the gerbil preproghrelin and glyceraldehyde-3-phosphate dehydrogenase homologue was isolated and a quantitative reverse transcription-polymerase chain reaction system was established.

Results: In gerbils showing $H$ pylori colonisation ( $H$ pylori group), expression of preproghrelin mRNA and total ghrelin levels were significantly decreased 17 and 23 weeks later $(p<0.01)$. Although the number of ghrelin immunoreactive cells decreased as the stomach weight increased, the gastric contents of total and active ghrelin in this group were the same as those in controls. Gastric myeloperoxidase activity showed a positive correlation with plasma ghrelin levels. On the other hand, at 17 weeks, plasma ghrelin levels were significantly increased in the $H$ pylori group $(p<0.05)$, suggesting a compensatory increase in secretion of the peptide at this time point.

Conclusion: The present experimental study demonstrated that gastric and plasma ghrelin dynamics are altered in response to $\mathrm{H}$ pylori infection.
\end{abstract}

$\mathrm{R}$ ecently, a novel growth hormone releasing peptide, ghrelin, was demonstrated to be localised mainly in the A-like cells of the gastric fundus. ${ }^{12}$ The rat ghrelin sequence was found to correspond to a 28 residue signal sequence within the 117 residue preproghrelin, which had also been elucidated previously in humans and mice. ${ }^{13-5}$ Ghrelin has been found to be of great importance in the regulation of appetite and weight control, as well as that of gastrointestinal functions, such as acid secretion and gastrointestinal motility. ${ }^{6}$

Helicobacter pylori (H pylori) infection is known to be a major pathogenetic factor in the development of gastritis, peptic ulcer disease, and gastric malignant lesions. ${ }^{89}$ Recently, Nwokolo and colleagues ${ }^{10}$ reported that the plasma ghrelin level increased after $H$ pylori eradicaton, suggesting a possible link between $H$ pylori infection and ghrelin secretion. In contrast, in another study, no significant difference in plasma ghrelin levels was reported between $H$ pylori positive and negative women of a similar age and body mass index. ${ }^{11}$ The results of two clinical studies were inconsistent, probably as they observed different aspects of ghrelin dynamics in $H$ pylori infection. To clarify the influence of $H$ pylori infection on gastric and plasma ghrelin dynamics directly, it would be necessary to conduct studies using established experimental animal models of $H$ pylori infection. The Mongolian gerbil is known to be a suitable animal model that shows marked gastric mucosal lesions in response to $H$ pylori infection; ${ }^{12-16}$ in contrast with the case in other experimental animals such as the mouse or rat. However, most of the nucleotide or amino acid sequences have not been identified in gerbils. We therefore characterised preproghrelin and glyceraldehyde-3phosphate dehydrogenase (GAPDH) in the Mongolian gerbil.

As it has been reported that ghrelin is produced and secreted from the gastric fundus ${ }^{17}{ }^{18}$ and that plasma ghrelin levels decrease significantly after gastrectomy, it is important to investigate the dynamics of ghrelin under diverse disease conditions of the gastric mucosa. The present study was designed to examine levels of gastric and plasma ghrelin in Mongolian gerbils with $H$ pylori colonisation of the gastric mucosa. ${ }^{13}{ }^{15}$ We found reduced levels of gastric ghrelin and a compensatory increase in plasma ghrelin levels after food deprivation in gerbils infected with $H$ pylori.

\section{METHODS}

\section{Cloning of gerbil ghrelin and GAPDH cDNA}

Total RNA was obtained from the Mongolian gerbil (Meriones unguiculatus) stomach using the RNeasy Mini-kit (Qiagen, Hilden, Germany). In order to clone a gerbil ghrelin homologue, degenerative primers were designed from human and mouse ghrelin CDNA (forward primer M01: 5'CCGGAATTCACTCAGCATGCTCTGGATGGACATGGCCATGGCAGG-3' and reverse primer M02: 5' - CCGGAATTCTCAGCTGGCGCCTCTTTGACCTCTTCCCA-3'). Approximately $2 \mu \mathrm{g}$ of total RNA were used as the template and one step reverse transcription-polymerase chain reaction (RT-PCR) was performed with the $\mathrm{M} 01$ and $\mathrm{M} 02$ primers $\left(50^{\circ} \mathrm{C}\right.$ for 30 minutes, $95^{\circ} \mathrm{C}$ for 15 minutes, and 35 cycles of $55^{\circ} \mathrm{C}$ for 30 seconds, $72^{\circ} \mathrm{C}$ for one minute, and $94^{\circ} \mathrm{C}$ for 20 seconds). The size of the PCR product was close to the expected size (332 bp); the product was subcloned into the pBluescript $\mathrm{SK}^{+}$vector (Stratagene, La Jolla, California, USA), and the DNA sequences were determined using the 310 Genetic Analyzer (PE Applied Biosystems, Norwalk, Connecticut, USA).

Abbreviations: GAPDH, glyceraldehyde-3-phosphate dehydrogenase; RT-PCR, reverse transcription-polymerase chain reaction; RIA, radioimmunoassay; $\mathrm{DAB}, 3,3^{\prime}$-diaminobenzidine tetrahydrochloride; $\mathrm{MPO}$, myeloperoxidase 
To obtain the full length cDNA of ghrelin, we performed 5'-RACE and 3'-RACE with primers designed based on the sequences obtained. For 5'-RACE, two gene specific antisense primers, M06 (5'-TCAGCTGGCGCCTCTTTGACCTCTT-3') and M07 (5'-GCTCCTGACAGCTTGATGCCAACGT-3'), were designed, and 5'-RACE was performed using the 5' RACE system for rapid amplification of the cDNA ends (Invitrogen Corp., Carlsbad, California, USA). For 3'-RACE, a gene specific sense primer, M05 (5'-TGGCCATGTCAGGCTCCAGCTTCTT-3'), was designed and $3^{\prime}$-RACE was performed using the SMART RACE cDNA Amplification Kit (BD Biosciences Clontech, Palo Alto, California, USA). Each amplified fragment was directly sequenced using the 310 Genetic Analyzer (PE Applied Biosystems).

For cloning of the gerbil homologue of GAPDH, the same method as that described above for ghrelin was used. In brief, to generate a fragment of GAPDH, forward primer M08 (5'CGGGGATCCGTATTGGGCGCCTGGTCACCAGGGC-3') and reverse primer Mll (5'-CCGGAATTCACTCCTTGGAGGCCATGTGGGCCAT-3') were used. The sizes of the PCR products were close to the expected size (990 bp). For $5^{\prime}$-RACE, a gene specific antisense primer, M17 (5'-CCTCAGTGTAGCCCAGGATGCCCTT-3'), was designed, and for 3'-RACE, a gene specific sense primer, Ml8 (5'-TCTTCACCACCATGGAGAAGGCCG-3'), was used. Each amplified fragment was directly sequenced using the 310 Genetic Analyzer (PE Applied Biosystems).

\section{Quantitative RT-PCR}

Total RNA was extracted from the stomach of the Mongolian gerbils using the RNeasy Mini Kit (Qiagen). A TaqMan quantitative real time RT-PCR was performed to detect preproghrelin mRNA and GAPDH mRNA with the ABI PRISM 7700 sequence detection system (PE Applied Biosystems). ${ }^{19}$

The following primers were used to amplify preproghrelin mRNA: ghrelin-F (5' -GGA ATC CAA GAA GCC ACC AGC-3'), ghrelin-R (5'-GCT CCT GAC AGC TTG ATG CCA-3'), and ghrelin-Taq (5'-FAM-AAC TGC AGC CAC GAG CTC TGG AAG GC-TAMRA-3'); to amplify GAPDH mRNA as the internal control, the following primers were used: GAPDHF (5'-TTC AAC GGC ACA GTC AAG GC-3'), GAPDH-R (5'GCC TTC TCC ATG GTG GTG AAG-3'), and GAPDH-Taq (5'FAM-CCC ATC ACC ATC TTC CAG GAG CGA GA-TAMRA-3').

The PCR fragments of the Mongolian gerbil preproghrelin and GAPDH were amplified and subcloned into the pDrive cloning vector (Qiagen) and respectively used as standards. Preproghrelin mRNA expression levels were normalised using GAPDH mRNA expression levels.

\section{Radioimmunoassay (RIA) for ghrelin}

\section{Stomach, duodenum, or jejunum sampling}

Fresh whole anterior wall specimens of the glandular stomach, duodenum (5-15 $\mathrm{mm}$ from the pyloric ring), or jejunum (120-130 $\mathrm{mm}$ from the pyloric ring) were frozen immediately after collection and stored at $-80^{\circ} \mathrm{C}$. Each sample was boiled for five minutes in a 10-fold volume of water to inactivate the intrinsic proteases. The solution was adjusted to $\mathrm{l} \mathrm{M}$ acetic acid after cooling, and the tissue was homogenised since acidification of sample solution prevented the loss of extraction recovery. The supernatant was lyophilised and then subjected to ghrelin RIA. The extraction efficiency of tissue ghrelin was more than 95\%.

\section{Plasma sampling}

Whole blood samples ( $1 \mathrm{ml}$ ) were obtained from the right ventricle in tubes containing EDTA-2Na ( $1 \mathrm{mg} / \mathrm{ml})$ and aprotinin $(500 \mathrm{kIU} / \mathrm{ml})$. Each plasma sample $(500 \mu \mathrm{l})$ was treated with a C18 Sep-Pak cartridge (Waters, Milford,
Massachusetts, USA) for peptide extraction. The cartridge was washed and eluted; the eluate $(50 \mu \mathrm{l}$ equivalent of plasma) was lyophilised and subjected to ghrelin RIA. The extraction efficiency of plasma ghrelin was approximately $90 \%$.

\section{RIA for ghrelin}

The two RIA techniques were used for measuring ghrelin, as described previously. ${ }^{20}$ Briefly, ghrelin levels were measured using two polyclonal rabbit antibodies raised against the $\mathrm{N}$ terminal (1-11) (Glyl-Lys11) or C terminal (13-28) (Gln13Arg28) fragment of rat ghrelin. Two tracer ligands were synthesised: [Tyr29]-rat ghrelin for antirat ghrelin (1-11) antiserum and [Tyr 12]-rat ghrelin (13-28) for antirat ghrelin (13-28) antiserum. RIA incubation mixtures, containing $100 \mu \mathrm{l}$ of either standard ghrelin or unknown sample with $200 \mu \mathrm{l}$ of antiserum diluted in RIA buffer containing $0.5 \%$ normal rabbit serum, were initially incubated for 12 hours. Then, $100 \mu \mathrm{l}$ of ${ }^{125} \mathrm{I}$ labelled tracer $(15000 \mathrm{cpm}$ ) were added and the mixture was incubated for 36 hours. Antirabbit IgG goat serum $(100 \mu \mathrm{l})$ was added prior to an additional 24 hour incubation period. Free and bound tracers were then separated by centrifugation at $3000 \mathrm{rpm}$ for 30 minutes. Following aspiration of the supernatant, radioactivity in the pellet was quantitated using a gamma counter (ARC-600; Aloka, Tokyo, Japan). All assays were performed at $4^{\circ} \mathrm{C}$. The antirat ghrelin (1-11) antiserum specifically recognised the $n$-octanoylated form of rat ghrelin but not the des-acyl form. The antirat ghrelin (13-28) antiserum recognised both the acylated and des-acyl forms of rat ghrelin equally. Both antisera were equally cross reactive with human and gerbil ghrelin and did not recognise the other enteric peptides. The respective intra- and interassay coefficients of variation for the $\mathrm{N}$ terminal RIA were $3 \%$ and $6 \%$, and for the $\mathrm{C}$ terminal RIA, $6 \%$ and $9 \%$.

\section{Mongolian gerbil model of $\boldsymbol{H}$ pylori infection}

All experiments and procedures carried out on animals were approved by the Keio University Animal Research Committee (No 023009). Thirty one specific pathogen free male Mongolian gerbils (MGS/Sea, five week old; Seac Yoshitomi, Fukuoka, Japan) were administered $H$ pylori suspensions (ATCC43504: $10^{8}$ colony forming units (CFU) $/ \mathrm{ml}$, $15 \mathrm{ml} / \mathrm{kg}$ ) while 27 control gerbils were administered buffer solution alone after overnight deprivation of food. All animals were allowed free access to water and a standard pellet diet (CE-2; Clea Japan, Tokyo, Japan). Four, 17, and 23 weeks after inoculation, the gerbils were examined under ether anaesthesia after 16 hours of food deprivation, and sacrificed by an overdose of ether.

$H$ pylori infection at each time point was examined by determining the number of CFU in a microaerobic bacterial culture. Briefly, the diluted homogenates of the stomachs were plated onto Brucella agar plates containing 10\% horse blood, $2.5 \mu \mathrm{g} / \mathrm{ml}$ amphotericin $\mathrm{B}, 9 \mu \mathrm{g} / \mathrm{ml}$ vancomycin, $0.32 \mu \mathrm{g} / \mathrm{ml}$ polymyxin $\mathrm{B}, 5 \mu \mathrm{g} / \mathrm{ml}$ trimethoprim, and $50 \mu \mathrm{g} / \mathrm{ml}$ 2, 3, 5-triphenyl-tetrazolium chloride. The plates were then incubated at $37^{\circ} \mathrm{C}$ in a microaerobic atmosphere for seven days. The number of colonies was counted, and the amount of viable $H$ pylori was expressed as the number of $\mathrm{CFU} / \mathrm{g}$ of tissue. ${ }^{21}$

\section{Immunohistochemistry}

Stomach tissue specimens of the gerbils were fixed in 10\% neutralised formalin and embedded in paraffin. Paraffin sections were placed on slides pretreated with a $0.01 \%$ aqueous solution of poly-L-lysine. Deparaffinisation and hydration were conducted. Then, the antigens were retrieved by heating for 15 minutes at $121^{\circ} \mathrm{C}$ in citrate buffer $(10 \mathrm{mM}$, 
$\mathrm{pH}$ 6.0). After cooling, endogenous peroxidase was quenched by $0.3 \%$ hydrogen peroxide. After washing, non-specific binding was blocked by a blocking reagent (BlockAce; Dainippon Pharm, Osaka, Japan). All sections were incubated overnight at $4^{\circ} \mathrm{C}$ with antighrelin (13-28) antiserum ( $1: 10000) .{ }^{17}$ After washing with TBS-T, slides were incubated with peroxidase labelled dextran polymer conjugated goat antimouse IgG in Tris-HCl (EnVision/HRP; Dako Japan, Kyoto, Japan) for 30 minutes at room temperature and then visualised after colour development using 3,3' -diaminobenzidine tetrahydrochloride (DAB) solution for three minutes. Counterstaining was performed with haematoxylin.

The stained sections were observed under a light microscope equipped with a 3CCD digital camera (C7780; Hamamatsu Photonics, Hamamatsu, Japan $)^{19}$ and each image was stored as a PSD file (Adobe Photoshop 7.0). Haematoxylin and/or DAB stained nuclei were counted using a particle analysis program (Ultimage Pro. 2.6.4; Alliance Vision, France). The density of ghrelin immunoreactive cells (D:ghrelin) was computed using the following equation:

$$
\text { D:ghrelin }=(\mathrm{Ng} / \mathrm{Nt}) \times 100(\%)
$$

where Ng and Nt represent the number of ghrelin immunoreactive cells and the total cell number, respectively, in the region of interest.

To investigate colocalisation of parietal cells with ghrelin immunoreactive cells, some specimens were also stained for $\mathrm{H}^{+}$-, $\mathrm{K}^{+}$-ATPase. After ghrelin immunostaining with DAB, specimens were incubated with a mouse anti- $\mathrm{H}^{+}-, \mathrm{K}^{+}$-ATPase alpha subunit monoclonal antibody (RDI, Flanders, New Jersey, USA; $1: 300$ ) overnight at $4^{\circ} \mathrm{C}$. The slides were then incubated with goat antimouse IgG conjugated to alkaline phosphatase labelled dextran polymer in Tris-HCl buffer (EnVision/AP) for 30 minutes at room temperature. Sections were then visualised after exposure to the Dako Fuchsin substrate chromogen system for seven minutes.

\section{MPO activity}

Tissue samples of gastric mucosa were collected in tubes containing phosphate buffered saline with protease inhibitors ( $100 \mu \mathrm{M}$ phenylmethylsulphonyl fluoride, $10 \mu \mathrm{g} / \mathrm{ml}$ aprotinin) and sonicated over ice in 30 consecutive 0.5 second bursts at 0.5 second intervals at a power setting of $150 \mathrm{~W}$ (VCX 750; Sonics and Materials, Inc., Newton, Connecticut, USA). Total protein in the homogenates was measured using the modified Lowry method, ${ }^{22}$ as described by Smith and colleagues. ${ }^{23}$

Myeloperoxidase (MPO) activity, an index of polymorphonuclear cell accumulation, was determined using a previously described method, with some modifications. ${ }^{24}$ Samples of mucosal homogenates were centrifuged at $8000 \mathrm{~g}$ for 15 minutes at $4^{\circ} \mathrm{C}$ to pellet the insoluble cellular debris. The pellet was then rehomogenised in an equal volume of $0.05 \mathrm{M}$ potassium phosphate buffer ( $\mathrm{pH}$ 5.4) containing $0.5 \%$ hexadecyltrimethylammonium bromide. Samples were centrifuged at $8000 \mathrm{~g}$ for 15 minutes at $4^{\circ} \mathrm{C}$ and the supernatants were saved. MPO activity was assessed by measuring $\mathrm{H}_{2} \mathrm{O}_{2}$ dependent oxidation of 3,3',5,5' -tetramethylbenzidine. One unit of enzyme activity was defined as the amount of MPO that caused a change in absorbance of 1.0/minute at $655 \mathrm{~nm}$ at $25^{\circ} \mathrm{C}$.

\section{Statistical analysis}

All data are expressed as mean (SEM); $p<0.05$ was considered to denote statistical significance. The data were analysed using one way analysis of variance followed by Scheffe's multiple comparison test.

\section{RESULTS}

cDNA and protein sequences of the Mongolian gerbil preproghrelin and characterisation of the peptide

The isolated full length Mongolian gerbil preproghrelin cDNA was $460 \mathrm{bp}$ long, consisting of $17 \mathrm{bp}$ in the $5^{\prime}$-untranslated region, $354 \mathrm{bp}$ of coding region, and $89 \mathrm{bp}$ in the $3^{\prime}$ untranslated region. The predicted initiation methionine was located at nucleotide position 18-20. An AATAAA polyadenylation signal was identified in the $3^{\prime}$ non-coding region (position 452-457) (fig lA) (Suzuki et al, EMBL/ GenBank: AF442491). The deduced amino acid sequence of the coding region indicated that gerbil preproghrelin was composed of 117 amino acid residues, the same length as that of the mouse, rat, and human preproghrelins (fig 1B) (AF442491). At the nucleotide level, the gerbil preproghrelin sequence exhibited an average homology score of $85 \%$ with human (AJ252278), 90\% with mouse (AJ243503), and 92\% with rat (AB029433) preproghrelins (fig 1B). The gerbil preproghrelin was highly conserved, the protein sharing $83 \%$ (97/117) identity with its human counterpart, 89\% (104/117) identity with its mouse counterpart, and $88 \%$ (103/117) identity with its rat counterpart. The unidentified third residue of the purified peptide was determined to be serine, the same as that in the case of other mammalian ghrelins. As gerbil ghrelin was determined to be entirely homologous with rat ghrelin apart from one amino acid $\left(\mathrm{Thr}^{12}\right)$, two polyclonal antibodies against rat ghrelin were used to measure the gerbil ghrelin in the present study.

\section{CDNA and protein sequence of the Mongolian gerbil GAPDH and characterisation of this peptide}

The isolated full length Mongolian gerbil GAPDH cDNA was $1265 \mathrm{bp}$ long, consisting of $71 \mathrm{bp}$ in the $5^{\prime}$-untranslated region, $1002 \mathrm{bp}$ in the coding region, and $192 \mathrm{bp}$ in the $3^{\prime}$ untranslated region (Suzuki et al, EMBL/GenBank: AY066007). The predicted initiation methionine was located at nucleotide position 72-74. The deduced amino acid sequence of the coding region indicated that the gerbil GAPDH may be composed of 333 amino acids residues, the same length as that of the mouse or rat GAPDH, and two amino acids shorter than the human GAPDH. The deduced amino acid sequence of gerbil GAPDH was 97.6\% (325/333) identical to that of its mouse counterpart, 97.3\% (324/333) identical to that of its rat counterpart, and 95.2\% (319/335) identical compared with its human counterpart.

\section{Preproghrelin mRNA expression and ghrelin contents in the stomach}

The level of preproghrelin mRNA as a ratio of the level of GAPDH mRNA (preproghrelin/GAPDH mRNA) in the $H$ pylori group was significantly decreased compared with that in controls, both at 17 and 23 weeks after $H$ pylori inoculation (fig 2).

Although there were no significant differences in total gastric and active ghrelin levels (fmol/mg) between the control and $H$ pylori groups at four weeks after inoculation, these levels were significantly decreased in the $H$ pylori group compared with those in the control group at both 17 and 23 weeks after inoculation (fig 3A, B).

\section{Ghrelin immunoreactive cells}

As shown in fig 4A, ghrelin immunoreactive cells were located in the mucosal neck glands of the gastric corpus, especially in the vicinity of $\mathrm{H}^{+}-, \mathrm{K}^{+}$-ATPase positive parietal cells, in non- $H$ pylori infected gerbils. Under higher magnification, ghrelin immunoreactive cells were found to be in contact with parietal cells in the same glands (fig 4B, C). In the gastric corpus mucosa of $H$ pylori colonised gerbils, although the density of $\mathrm{H}^{+}-, \mathrm{K}^{+}$-ATPase positive cells was 


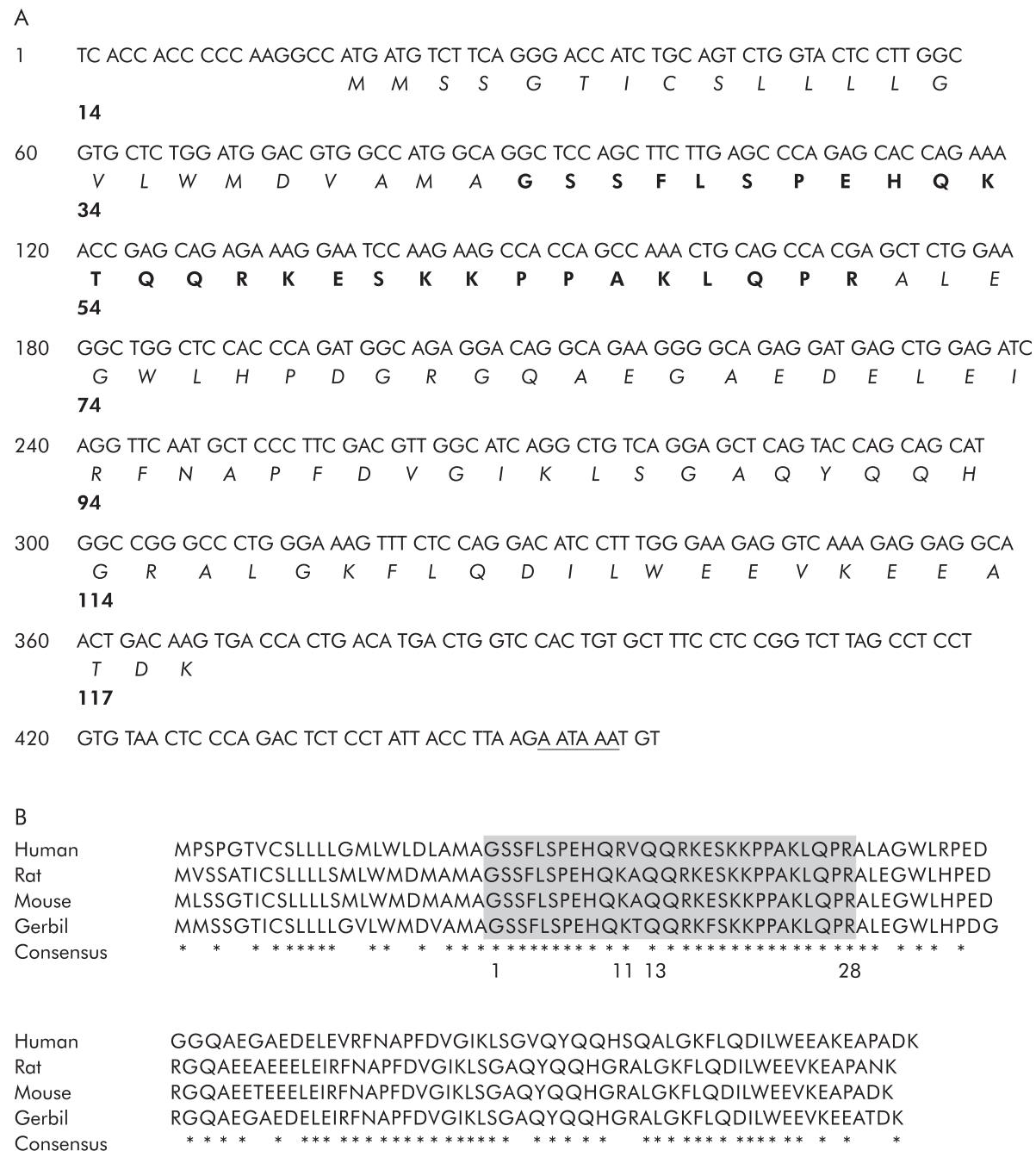

Figure 1 CDNA and protein sequences of preproghrelin and their homology with those of other species. (A) Nucleotides and amino acids are numbered on the left and right, respectively. The amino acid residues in the signal peptide sequence are printed in italics. The amino acid residues constituting the matured ghrelin peptides are printed in bold type. The EMBL/GenBank accession number for this sequence is AF442491. (B) Alignment of human, rat, mouse, and Mongolian gerbil preproghrelin protein sequences. Matching residues are indicated as homologous (*). Amino acids corresponding to the ghrelin peptide are shown as the shaded area.

decreased in association with accumulation of inflammatory cells, ghrelin immunoreactive cells were still found to be localised in association with these $\mathrm{H}^{+}-, \mathrm{K}^{+}$-ATPase positive cells (fig 4D). On the other hand, in H pylori colonised corpus mucosa showing severe atrophy, extensive disappearance of

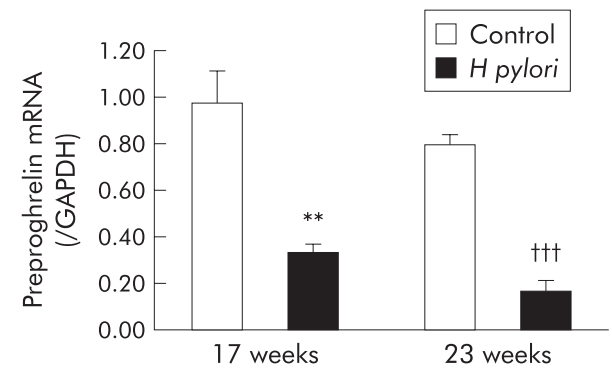

Figure 2 Preproghrelin mRNA expression levels normalised to glyceraldehyde-3-phosphate dehydrogenase (GAPDH) mRNA expression levels in the stomach of Mongolian gerbils. ${ }^{* *} \mathrm{p}<0.01$ compared with control at 17 weeks; $\uparrow+\uparrow p<0.001$ compared with controls at 23 weeks.
$\mathrm{H}^{+}-, \mathrm{K}^{+}$-ATPase positive cells was noted and the number of ghrelin immunoreactive cells was also decreased (fig 4E).

Representative photomicrographs of ghrelin immunohistochemistry in the corpus revealed a decrease in the number of brown stained ghrelin immunoreactive cells in $H$ pylori colonised mucosa (fig 5B, D).

Morphometeric analysis also revealed that the number of ghrelin immunoreactive cells $/ \mathrm{mm}^{2}$ was significantly decreased in $H$ pylori colonised mucosa of gastric corpus, both at 17 and 23 weeks after $H$ pylori inoculation (table 1). In contrast, the total cell number was found to be higher in $H$ pylori colonised mucosa (table 1), possibly due to inflammatory cell infiltration. Taking these parameters into consideration, it was found that the density of ghrelin immunoreactive cells (\%) was decreased in $H$ pylori infected gastric corpus mucosa (table 1), suggesting a possible relationship between the increase in the number of inflammatory cells and decrease in the number of ghrelin immunoreactive cells.

\section{Gastric mucosal inflammation}

During the observation period, persistent $H$ pylori colonisation was confirmed in all $H$ pylori inoculated gerbils. No significant difference in body weight was observed between 

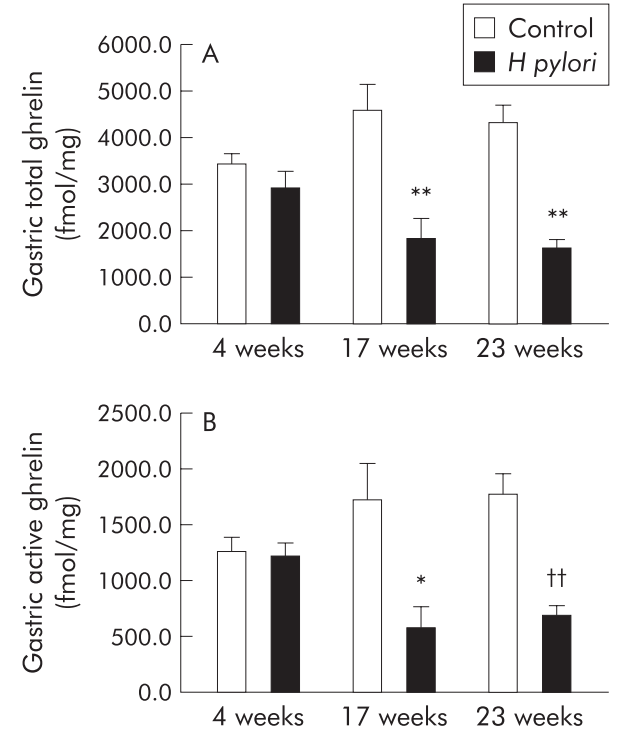

Figure 3 Levels of ghrelin in the stomach of Mongolian gerbils. (A) Total ghrelin levels in the stomach of Mongolian gerbils with or without Helicobacter pylori colonisation at 17 and 23 weeks after bacterial inoculation. ${ }^{* *} p<0.01$ compared with controls at each of the time points. (B) Active ghrelin levels in the stomach of Mongolian gerbils with or without $H$ pylori colonisation at 17 and 23 weeks after bacterial inoculation. ${ }^{*} \mathrm{p}<0.05$ compared with controls at 17 weeks; $\uparrow+p<0.01$ compared with controls at 23 weeks.

the control and $H$ pylori groups (table 2), suggesting that although $H$ pylori altered the ghrelin dynamics, it probably did not influence the eating behaviour or body mass of the animals in such a short period of time after the onset of infection. In this situation, some compensatory mechanism may be evoked to regulate ghrelin independent growth hormone secretion and body mass. In contrast, stomach wet weight was significantly increased in the $H$ pylori group (table 2), which could reflect the macroscopic findings of severe thickening and oedematous changes associated with severe inflammation observed in $H$ pylori colonised gastric mucosa. Consistent with the increase in stomach weight, gastric mucosal MPO activity, which reflects polymorphonuclear infiltration, was increased significantly compared with that in controls 17 and 23 weeks after $H$ pylori inoculation (table 2). At four weeks after inoculation, although the wet weight of the stomach was significantly increased in the $H$ pylori group (table 2), no significant increase in MPO activity was observed, reflecting the merely oedematous changes of the gastric mucosa in the acute phase of infection.

Notwithstanding the above data on stomach weight, total and active ghrelin levels in each whole stomach specimen were determined to be the same in the control and $H$ pylori groups (table 3 ).

\section{Plasma ghrelin levels}

Although the fasting plasma total ghrelin level was significantly increased in the $H$ pylori group compared with that of controls at 17 weeks after $H$ pylori inoculation, the difference was not significant at four or 23 weeks (fig 6A). In contrast, fasting plasma active ghrelin levels were significantly increased in the $H$ pylori group at both 17 and 23 weeks after $H$ pylori inoculation compared with those in each control group, although no significant increase in plasma active ghrelin level was shown at four weeks (fig 6B).

Gastric mucosal MPO activity was correlated with plasma levels of total and active ghrelin (fig 7).
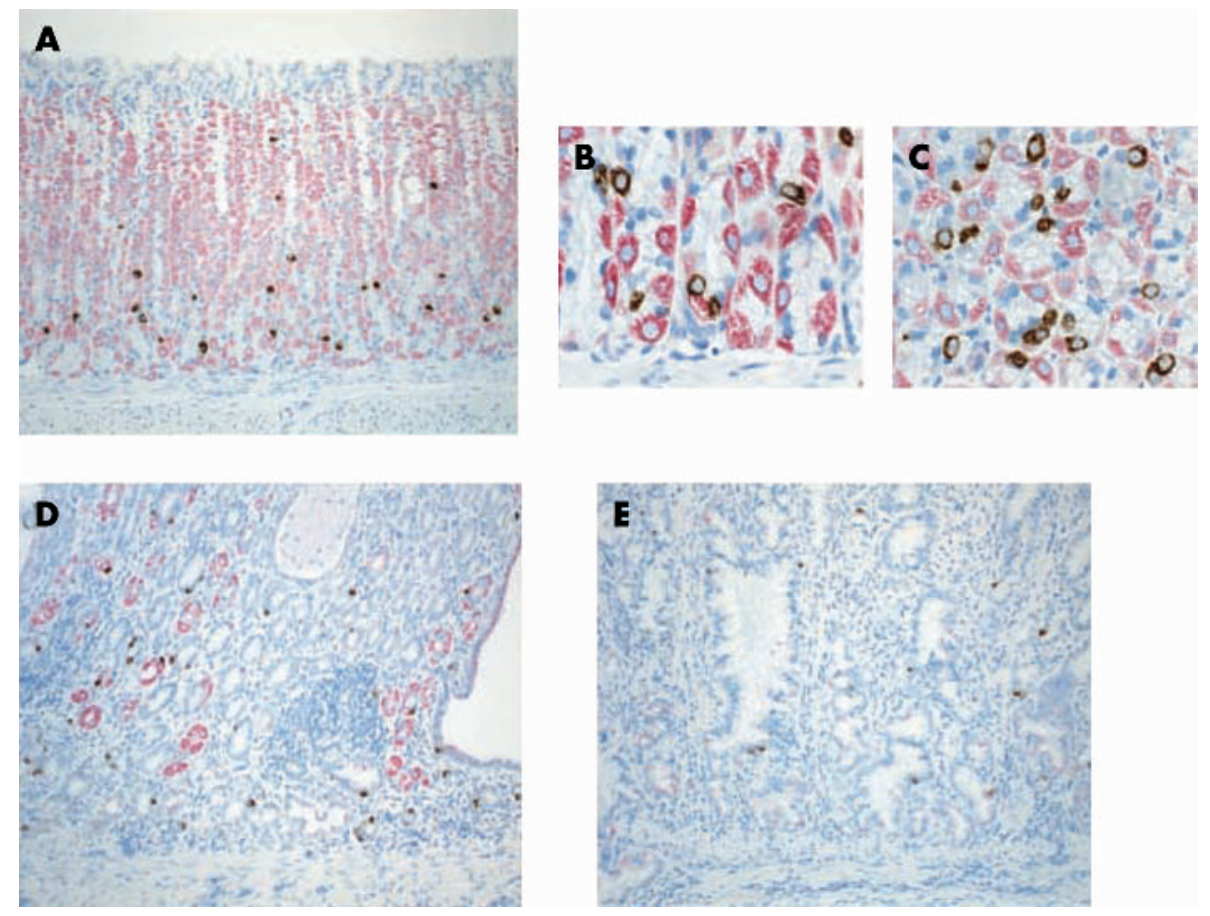

Figure 4 Colocalisation of ghrelin immunoreactive cells with parietal cells in the gastric fundic mucosa of Mongolian gerbils. The brown coloured cells represent ghrelin immunoreactive cells and the pink coloured cells represent $\mathrm{H}^{+}, \mathrm{K}^{+}$-ATPase positive parietal cells. (A) Control fundic mucosa; magnification $20 \times$. (B) Control fundic mucosa; magnification $100 \times$, longitudinal view. (C) Control fundic mucosa; magnification $100 \times$, cross sectional view. (D) Helicobacter pylori colonised fundic mucosa showing inflammatory cell infiltration; magnification $20 \times$. (E) $H$ pylori colonised fundic mucosa showing severe gastric mucosal atrophy; magnification $20 \times$. 

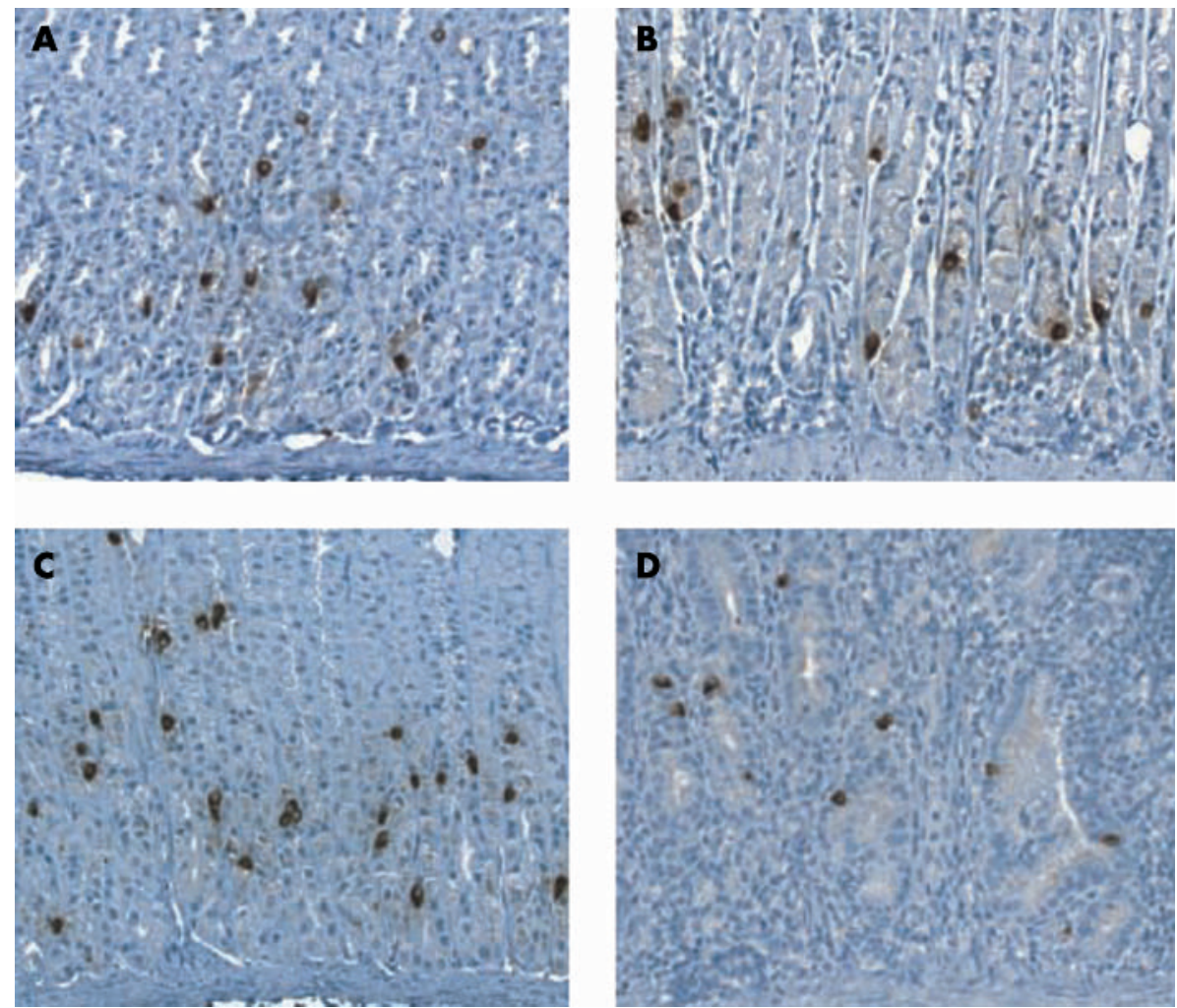

Figure 5 Representative photomicrograph of ghrelin immunoreactive cells in the gastric fundic mucosa of Mongolian gerbils. The brown coloured cells represent ghrelin immunoreactive cells. (A) Control fundic mucosa at 17 weeks after Helicobacter pylori inoculation. (B) $H$ pylori colonised fundic mucosa at 17 weeks after $\mathrm{H}$ pylori inoculation. (C) Control fundic mucosa at 23 weeks after $\mathrm{H}$ pylori inoculation. (D) $\mathrm{H}$ pylori colonised fundic mucosa at 23 weeks after $H$ pylori inoculation; magnification $40 \times$.

\section{Ghrelin levels in the duodenum and jejunum}

Figure 8 demonstrates levels of total ghrelin in the stomach, duodenum, and jejunum in both the control and $H$ pylori groups at four weeks after $H$ pylori inoculation. Although no significant difference in the content of ghrelin in any part of the intestine was observed between the control and $H$ pylori groups at this time point, levels were significantly reduced to $0.55 \%$ and $0.16 \%$ in the duodenum and jejunum, respectively, compared with stomach levels, suggesting predominant localisation of ghrelin in the stomach of gerbils.

\section{DISCUSSION}

Modified ghrelin dynamics in $H$ pylori infection were demonstrated in the present study using an experimental animal model, and reduced preproghrelin mRNA expression and ghrelin levels were clearly shown in the stomach of gerbils with $H$ pylori colonisation, in association with a significant increase in inflammatory cell infiltration.

The amino acid sequence corresponding to the ghrelin peptide in the gerbil was $93 \%$ identical to that of humans and $96 \%$ identical to that of its mouse or rat counterpart (fig 1B),

Table 1 Ghrelin immunoreactive cells in the fundic mucosa of Mongolian gerbils

\begin{tabular}{|c|c|c|c|c|}
\hline & \multicolumn{2}{|l|}{17 weeks } & \multicolumn{2}{|l|}{23 weeks } \\
\hline & Control $(n=9)$ & $H$ pylori $(n=6)$ & Control $(n=12)$ & $H$ pylori $(n=11)$ \\
\hline $\begin{array}{l}\text { Ghrelin immunoreactive cells (No/0.1 } \mathrm{mm}^{2} \text { ) } \\
\left.\text { Total cell number (No/0.1 mm }{ }^{2}\right) \\
\text { Ghrelin immunoreactive cells (\%) }\end{array}$ & $\begin{array}{c}16.1(1.0) \\
677.2(21.4) \\
2.4(0.1)\end{array}$ & $\begin{array}{r}10.1(1.0)^{*} \\
792.3(15.0) \\
1.3(0.1)^{\star *}\end{array}$ & $\begin{aligned} 17.3 & (0.8) \\
681.5 & (12.8) \\
2.6 & (0.1)\end{aligned}$ & $\begin{array}{c}12.3(0.7) \dagger \dagger \\
772.6(35.6) \\
1.7(0.1) \dagger \dagger\end{array}$ \\
\hline
\end{tabular}

Table 2 Body weight, stomach weight, and gastric myeloperoxidase (MPO) activity of Mongolian gerbils

\begin{tabular}{|c|c|c|c|c|c|c|}
\hline & \multicolumn{2}{|l|}{4 weeks } & \multicolumn{2}{|l|}{17 weeks } & \multicolumn{2}{|l|}{23 weeks } \\
\hline & Control $(n=10)$ & $H$ pylori $(n=10)$ & Control $(n=9)$ & $H$ pylori $(n=6)$ & Control $(n=12)$ & $H$ pylori $(n=11)$ \\
\hline Body weight (g) & $58.3(0.7)$ & $55.5(0.8)$ & $76.9(2.2)$ & $74.5(1.4)$ & $79.0(1.9)$ & $84.1(4.1)$ \\
\hline Wet weight of stomach $(\mathrm{mg})$ & $632.1(14.1)$ & $765.2(53.9)^{*}$ & $619.1(16.2)$ & $1172.8(28.5) \dagger+\dagger$ & $613.6(16.1)$ & 1126.1 (42.2)㧊 \\
\hline $\begin{array}{l}\text { Gastric MPO activity } \\
\text { (mU/mg protein) }\end{array}$ & $0.9(0.2)$ & $0.2(0.1)$ & $0.6(0.1)$ & $6.9(0.6)^{*}$ & $0.4(0.1)$ & 4.2 (0.8)㧊 \\
\hline
\end{tabular}


Table 3 Ghrelin contents (nmol) per one stomach in Mongolian gerbils

\begin{tabular}{|c|c|c|c|}
\hline \multicolumn{2}{|l|}{17 weeks } & \multicolumn{2}{|l|}{23 weeks } \\
\hline Control $(n=9)$ & $H$ pylori $(n=6)$ & Control $(n=12)$ & $H$ pylori $(n=11)$ \\
\hline $\begin{array}{l}2.91(0.38) \\
1.10(0.46)\end{array}$ & $\begin{array}{l}2.14(0.48) \\
0.67(0.19)\end{array}$ & $\begin{array}{l}2.71(0.29) \\
1.12(0.64)\end{array}$ & $\begin{array}{l}2.20(0.32) \\
1.00(0.78)\end{array}$ \\
\hline
\end{tabular}

suggesting functional homogeneity of this peptide in various animals. Differences in the amino acid sequences among the four species examined were found only at position 34 or 35 (fig 1B), a rather small segment of the molecule, probably insignificant in relation to biological function. ${ }^{3}$ Indeed, studies on the structure-activity relationship of the peptide showed that the octanoyl group on $\mathrm{Ser}^{3}$ is the essential moiety for biological activity of this peptide, ${ }^{1}$ and that the $\mathrm{N}$ terminal fragments showing conservation of the first five amino acids exhibited full functional activity. ${ }^{25}$

Using the same experimental animal, we previously reported a significant increase in the extent of inflammatory cell infiltration and oxidative stress in $H$ pylori colonised gastric mucosa, ${ }^{15}$ which validated the usefulness of this model for the study of $H$ pylori associated gastritis. The present study also showed marked gastric inflammation induced following $H$ pylori inoculation (table 2), which was reflected by an increase in stomach weight (table 2 ).

As ghrelin levels in the whole stomach specimens with $H$ pylori infection were in the same range as those in controls, even after release of the peptide into the bloodstream in response to food deprivation (table 3), it would be difficult to propose that the increase in plasma ghrelin levels in gerbils with $H$ pylori infected gastric mucosa (fig 6) could be the result of increased gastric total ghrelin production. Increase in plasma ghrelin levels, particularly active ghrelin, may be
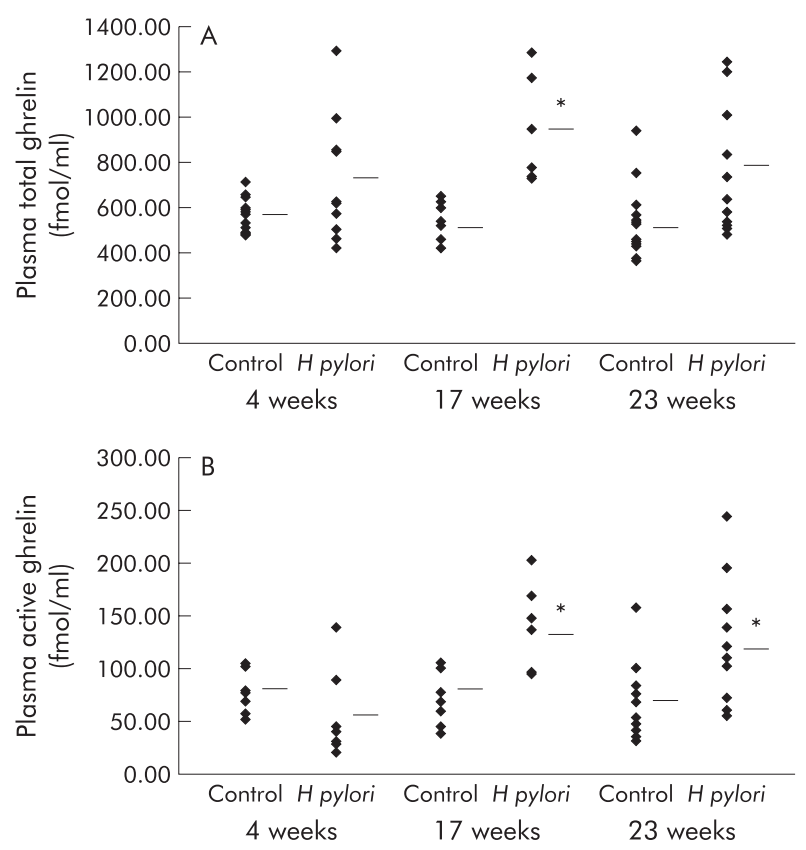

Figure 6 Plasma levels of ghrelin in Mongolian gerbils. (A) Total plasma ghrelin levels in Mongolian gerbils with or without Helicobacter pylori colonisation at 17 and 23 weeks after $\mathrm{H}$ pylori inoculation. ${ }^{*} p<0.05$ compared with controls at 17 weeks. (B) Plasma active ghrelin levels in Mongolian gerbils with or without $H$ pylori colonisation at 17 and 23 weeks after $H$ pylori inoculation. ${ }^{*} p<0.05$ compared with controls at the time points indicated. due to enhancement of degranulation of the ghrelin producing A-like cells induced by inflammatory stimuli, such as cytokines ${ }^{26}$ or free radicals. ${ }^{15}$ As plasma ghrelin elevation has been reported to occur in association with an increase in tumour necrosis factor $\alpha,{ }^{27}$ the increase in $H$ pylori associated tumour necrosis factor $\alpha$ production ${ }^{28}$ may play a role in the enhanced ghrelin release noted following food deprivation. These contentions are endorsed by the significant correlation between gastric MPO activity (table 1) and plasma ghrelin levels (fig 7). Even though the underlying mechanisms have still to be elucidated in detail, there may be a compensatory increase in levels of plasma ghrelin following food deprivation in $H$ pylori colonised gerbils, in association with a decrease in gastric ghrelin density. Such elevation of plasma ghrelin levels may attenuate the reduction in appetite and
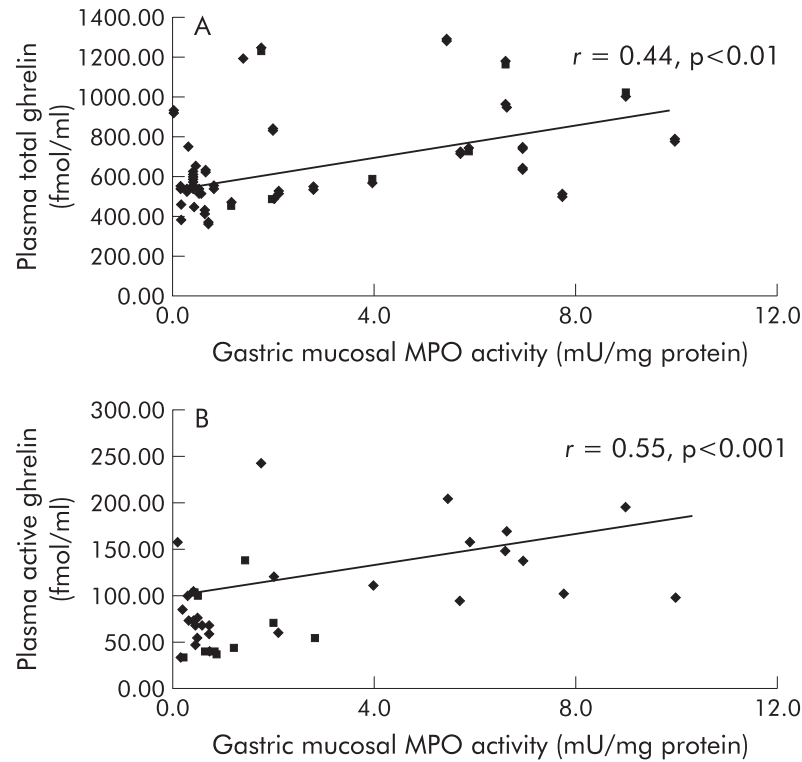

Figure 7 Relationship between gastric myeloperoxidase (MPO) activity and plasma ghrelin level. (A) Gastric mucosal MPO activity was well correlated with total plasma levels of ghrelin. (B) Gastric mucosal MPO activity was well correlated with plasma levels of active ghrelin.

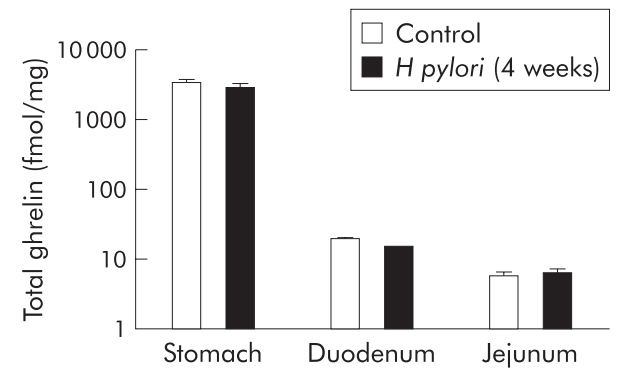

Figure 8 Ghrelin contents in the glandular stomach, duodenum, and jejunum, four weeks after Helicobacter pylori inoculation (fmol/mg). 
body weight in $H$ pylori infected gerbils until 23 weeks (table 2). As longer term $H$ pylori infection ( 72 weeks) than in the cohorts examined in the present study ( 23 weeks) reportedly evoked significant weight loss in gerbils, ${ }^{29}$ further extension of gastric inflammation and atrophy may nullify the effects of the compensatory increase in plasma ghrelin levels and lead to reduction of both appetite and body weight.

As the average intestinal wet weight in uninfected gerbils at four weeks after inoculation was $1540 \mathrm{mg}$, the total ghrelin content in the small intestine was computed to be less than $0.029 \mathrm{nmol}$ (fig 8). In contrast, the average wet weight of the stomach in uninfected gerbils at four weeks was $632.1 \mathrm{mg}$ (table 2) and the average gastric total ghrelin content was computed to be $2.168 \mathrm{nmol}$. The gastric total ghrelin content was estimated to be at least 74 -fold greater than that of the small intestine. Therefore, it would be difficult to consider an extragastric source of ghrelin as the origin of the increased plasma ghrelin in $H$ pylori colonised gerbils.

The present data in gerbils were inconsistent with the results of two recent clinical studies, ${ }^{10}{ }^{11}$ the results of which were also discrepant. These discrepancies might be attributable to differences in the methods of measurement of ghrelin peptide or patient selection, or interspecies difference in the distribution of extragastric ghrelin.

In the present study, plasma total ghrelin levels were not significantly increased in $H$ pylori colonised cohorts at 23 weeks after $H$ pylori inoculation (fig 6). Among the $H$ pylori colonised cohorts at 23 weeks, four of 11 gerbils showed plasma total ghrelin levels below the average value observed in controls at the corresponding time point. Among these four gerbils with $H$ pylori infection, two showed severe gastric atrophy, with the number of ghrelin immunoreactive cells being less than $1.2 \%$, as well as lower than average values for gastric ghrelin and preproghrelin mRNA. Severe atrophy might account for the absence of a statistically significant difference between infected and uninfected gerbils at 23 weeks. These results suggest that the ghrelin content in the stomach decreases in response to $H$ pylori infection, and with further extension of gastric atrophy, plasma ghrelin levels also decrease. Although there does seem to be differential regulation of preproghrelin mRNA, mucosal ghrelin, and possibly secretion of active ghrelin, the molecular physiology underlying ghrelin dynamics is outside the purview of the current study. Further investigation of ghrelin dynamics based on long term $H$ pylori infection could help to clarify these issues.

\section{ACKNOWLEDGEMENTS}

This study was supported by a Grant-in-Aid for Scientific Research C (2) from the Japan Society for the Promotion of Science (JSPS) (15590686, to HS), and a grant from Keio University School of Medicine. The authors thank Miss Kumiko Kurabayashi, Centre for Integrated Medical Research, Keio University School of Medicine, and Dr Hisashi Hisatomi, Centre for Molecular Biology and Cytogenetics, SRL, Inc, for their technical support.

\section{Authors' affiliations \\ H Suzuki, T Masaoka, H Ishii, Department of Internal Medicine, School of Medicine, Keio University, Tokyo, Japan \\ H Hosoda, K Kangawa, National Cardiovascular Centre Research Institute, Osaka, Japan \\ T Ota, Department of Dermatology, School of Medicine, Keio University, Tokyo, Japan}

Y Minegishi, S Nomura, Centre for Integrated Medical Research (4N9), School of Medicine, Keio University, Tokyo, Japan

\section{REFERENCES}

1 Kojima M, Hosoda H, Date $Y$, et al. Ghrelin is a growth-hormone-releasing acylated peptide from stomach. Nature 1999;402:656-60.

2 Bowers CY. Unnatural growth hormone-releasing peptide begets natural ghrelin. J Clin Endocrinol Metab 2001;86:1464-9.

3 Hosoda H, Kojima M, Matsuo $H$, et al. Purification and characterization of rat des-Gln14-Ghrelin, a second endogenous ligand for the growth hormone secretagogue receptor. J Biol Chem 2000;275:21995-2000.

4 Tanaka M, Hayashida Y, Iguchi T, et al. Organization of the mouse ghrelin gene and promoter: occurrence of a short noncoding first exon. Endocrinology $2001 ; 142: 3697-700$.

5 Coulie BJ, Miller U. Identification of motilin-related peptide. Gastroenterology 2001; 120:588-9.

6 Tomasetto C, Karam SM, Ribieras S, et al. Identification and characterization of a novel gastric peptide hormone: the motilin-related peptide. Gastroenterology 2000; 1 19:395-405.

7 Invi A. Ghrelin: an orexigenic and somatotrophic signal from the stomach. Nat Rev Neurosci 2001;2:551-60.

8 Marshall BJ, Warren JR. Unidentified curved bacilli in the stomach of patients with gastritis and peptic ulceration. Lancet 1984;1:1311-15.

9 Uemura N, Okamoto S, Yamamoto S, et al. Helicobacter pylori infection and the development of gastric cancer. N Engl J Med $2001 ; 345: 784-9$.

10 Nwokolo CU, Freshwater DA, O'Hare P, et al. Plasma ghrelin following cure of Helicobacter pylori. Gut 2003;52:637-40.

11 Gokcel A, Gumurdulu Y, Kayaselcuk F, et al. Helicobacter pylori has no effect on plasma ghrelin levels. Eur J Endocrinol 2003;148:423-6.

12 Yokota K, Kurebayashi Y, Takayama Y, et al. Colonization of Helicobacter pylori in the gastric mucosa of Mongolian gerbils. Microbiol Immunol 1991;35:475-80.

13 Hirayama F, Takagi S, Kusuhara $\mathrm{H}$, et al. Induction of gastric ulcer and intestinal metaplasia in Mongolian gerbils infected with Helicobacter pylori. J Gastroenterol 1996;31:755-7.

14 Matsumoto S, Washizuka Y, Matsumoto $Y$, et al. Induction of ulceration and severe gastritis in Mongolian gerbil by Helicobacter pylori infection. J Med Microbiol 1997;46:391-7.

15 Suzuki H, Mori M, Seto K, et al. H.pylori-associated gastric pro- and antioxidant formation in Mongolian gerbils. Free Radic Biol Med 1999;26:679-84.

16 Watanabe T, Tada M, Nagai $\mathrm{H}$, et al. Helicobacter pylori infection induces gastric cancer in Mongolian gerbils. Gastroenterology 1998;1 15:642-8.

17 Date Y, Kojima M, Hosoda H, et al. Ghrelin, a novel growth hormonereleasing acylated peptide, is synthesized in a distinct endocrine cell type in the gastrointestinal tracts of rats and humans. Endocrinology 2000;141:4255-61.

18 Ariyasu H, Takaya K, Tagami T, et al. Stomach is a major source of circulating ghrelin, and feeding state determines plasma ghrelin-like immunoreactivity levels in humans. J Clin Endocrinol Metab 2001;86:4753-8.

19 Masaoka T, Suzuki H, Hosoda H, et al. Enhanced plasma ghrelin levels in rats with streptozotocin-induced diabetes. FEBS Lett 2003;541:64-8.

20 Hosoda H, Kojima M, Matsuo H, et al. Ghrelin and des-acyl ghrelin: two major forms of rat ghrelin peptide in gastrointestinal tissue. Biochem Biophys Res Commun 2000;279:909-13.

21 Takahashi S, Keto Y, Fujita H, et al. Pathological changes in the formation of Helicobacter pylori-induced gastric lesions in Mongolian gerbils. Dig Dis Sci 1998:43:754-65.

22 Lowry OH, Rosebrough NJ, Farr AL, et al. Protein measurement with the Folin phenol reagent. J Biol Chem 1951;193:265-75.

23 Smith PK, Krohn RI, Hermanson GT, et al. Measurement of protein using bicinchoninic acid. Anal Biochem 1985;150:76-85.

24 Grisham MB, Hernandez LA, Granger DN. Xanthine oxidase and neutrophil infiltration in intestinal ischemia. Am J Physiol 1986;252:G567-74.

25 Bednarek MA, Feighner SD, Pong SS, et al. Structure-function studies on the new growth hormone-releasing peptide, ghrelin: minimal sequence of ghrelin necessary for activation of growth hormone secretagogue receptor la. J Med Chem 2000;43:4370-6.

26 Yamamoto N, Sakagami T, Fukuda Y, et al. Influence of Helicobacter pylori infection on development of stress-induced gastric mucosal injury. J Gastroenterol 2000;35:332-40.

27 Nagaya N, Uematsu M, Kojima M, et al. Elevated circulating level of ghrelin in cachexia associated with chronic heart failure: relationships between ghrelin and anabolic/catabolic factors. Circulation 2001;104:2034-8.

28 Crabtree JE, Shallcross TM, Heatley RV, et al. Mucosal tumour necrosis factor alpha and interleukin- 6 in patients with Helicobacter pylori associated gastritis. Gut 1991;32:1473-7.

29 Keto Y, Ebata M, Okabe S. Gastric mucosal changes induced by long term infection with Helicobacter pylori in Mongolian gerbils: effects of bacteria eradication. J Physiol (Paris) 2001;95:429-36. 